\title{
SOME PROBLEMS OF THE RHEUMATIC CHILD.
}

\author{
$B y$ C. E. SUNDELL, M.D., M.R.C.P.
}

(Pkysician to Seamen's Hospital, Greenwich. Consulting Physician to Evelina Hospital and Children's Department, Prince of Wales' Hospital.)

Confronted by a child suffering from rheumatism we must relegate to second place consideration of the interesting but vexed question whether this is due to streptococcal infection or to a fault in physiological chemistry. We need rather to form some clear conception of the actual processes at work, how their dangers may be recognised and forestalled, how we may gauge the success or failure of our efforts towards these ends and in the former case when we may sound the " all clear." Supplementary to this is the further question of prevention of further attacks.

In this connection it is advisable to consider for a moment the disputed existence of a rheumatic type of child, i.e. a physical and psychological mould of child-life that can be recognised as a precursor or concomitant of rheumatism. Our forefathers had no doubts of the existence of such types: they recognised two forms, one, fair or red-haired, freckling, delicate-skinned, sensitive, friendly, mentally and physically alert, easily moved to laughter or tears, docile and companionable ; the other coarser-grained in character, skin and feature, tending to sullenness, sometimes vindictive, passionate and surly. This latter type is fortunately the less common but they are both easily recognised, and a moment's reflection upon those children who have been our own patients will confirm the opinion of our medicab forebears. When they are encountered it is well to bear in mind their innate predisposition to rheumatism. They represent examples of "constitution" whiclo. is apt to be ignored at the present day but whose recognition and study are welF worth while.

There can be no doubt that children presenting the characters just described are, under the stress of harmful influences, more prone than others to develop rheumatic troubles of all kinds.

Such malign influences are malnutrition, exposure to damp, excessive or inadequate clothing or, what is more common, failure to adjust the warmth and dryness of clothing to changing atmospheric conditions, inadequate sleep, insufficient food or an ill-adjusted diet with a relative excess of sugars and starches and deficiency of fats, fruit and vegetables. Recognition of inborn vulnerability to such adverse conditions will point the way to preservation of the likely victim from rheumatism.

These considerations apply chiefly to frank acute rheumatic fever in childhood 9 but they apply also to less dramatic conditions such as stiff-neck, growing pains, repeated sore throats and the tendency to recurrent catarrhs of the upper respiratory tract which, like chorea, may for a time be the only evidence of an underlying $N$ rheumatic state liable to flare up into rheumatic fever.

Every case of rheumatic fever presents in some measure the familiar features of fever, sweating, joint-pains and joint-swelling. This picture varies in details with the age of the patient. In gross form it is rare under the age of seven years but it can occur as early as three years. Generally it may be said that the younger $\overbrace{0}$ the child the less severe and less lasting are the joint-changes. In a young child $\stackrel{?}{\rightarrow}$ the characteristic flitting of pain and swelling from joint to joint may not be seen; one joint only may be affected and that but for a short time. In every case irrespective of age, however, the triad of pain, fever and sweating is to be found. 
It is of interest to consider whether this combination of fever and sweating is a symptom-complex of the disease itself or whether it may not be a helpful reaction of the body to the disease. If the former, efforts to overcome it are justifiable and useful; if the latter, they might be unjustifiable and harmful. There is some evidence in favour of the view that fever and sweating represent a beneficial or curative reaction of the body.

Before the introduction of salicylate-treatment rheumatism was regarded as a self-limiting disease which ran its course in about six weeks and then cleared up without sequelæ. Gee's cases successfully treated with peppermint water are classical. Encouragement of sweating and maintenance of a raised temperature by means of hot-packs, blanket-baths or the thermal couch has undoubtedly speeded up recovery without salicylates and has proved of special value in clearing up those tedious cases in which a subacute rheumatic state lingers on after the acute manifestations have subsided. (Sundell, I928.)

Salicylates will control fever and relieve joint pains and swelling to a most welcome degree but it is possible that such relief may be too dearly bought at the price of failure to eradicate the underlying state which has produced these symptoms. In the case of a child racked by rheumatic pain salicylates must be used but they should be discontinued as soon as pain is relieved: when this result has been achieved they should not be pushed till the temperature falls to normal and stays there. Such a result may be long in coming and meanwhile the curative process of fever and sweating is being hampered in its effort to rid the child of a dangerous rheumatic state. There is no evidence to show that salicylates have ever prevented a cardiac lesion or exerted any control upon it when once established. Fear of heart-trouble need cause no hesitation in being very sparing in their use or in withholding them altogether.

Rheumatism as has already been said is a self-limiting disease: return of the joints to a healthy painless state is usually rapid and complete but in the adolescent some degree of chronic inflammatory arthritis occasionally follows the partial clearing-up of the acute condition. Over this salicylates appear to exert no influence; eradication of possible septic foci in tonsil, sinus or middle-ear is rarely successful. Cure follows the re-establishment of nature's method of resistance by fever and sweating in the ways already mentioned. (Sundell, I927.)

That heart-affection may occur is the fear of the doctor in every case of rheumatic disease in childhood.

It has been shown that in the majority of cases of acute rheumatism in children there is an alteration of the electrocardiogram pointing to some degree of heartinvolvement. It is, therefore, a wise plan to regard every case as complicated by heart-changes till we can convince ourselves that such changes do not exist.

$\mathrm{X}$-ray examination may reveal enlargement of the heart-shadow but in estimating this allowance must be made for the fact that the child is lying flat and not in the upright position usual for such an investigation.

In the absence of such technical facilities reliance must be placed upon ordinary clinical observations. By such measures carried out conscientiously and repeatedly it is possible to find the answer to the question-is the heart involved ?

The position of the visible and tangible apex-beat is not a completely reliable criterion. In the healthy young child it is often to be found in the nipple line. 
Enlargement of the area of cardiac duilness on percussion unless gross is difflcult to detect. One examination will rarely give information that might not be surmised from other signs but the finding of variations in size from week to week is of great significance.

If previous examinations have disclosed normal clear heart-sounds the appearance of even a slight transient systolic murmur at the apex or between the apex and the sternum is conclusive of a heart-lesion. It spells either dilatation or an early valvular lesion.

Pulse rate is a useful indication of the heart condition if it be remembered that the young child's heart is easily quickened by excitement and that if the temperature is raised an allowance of 8-ro beats per minute must be made for each rise of I degree Fahrenheit.

Pulse-rhythm is important: gross arrhythmia or extra-systoles will in a child bespeak heart-affection. Vagal or juvenile arrhythmia (alternate slowing and quickening of the heart-beat coincident with deep breathing) is strong presumptive evidence that the heart is healthy. This sign is best observed when there is no tachycardia and its significance is greatest when it returns during recovery after a period of abeyance.

In addition to the foregoing there are certain observations of a more general nature that may be helpful.

Pallor, particularly if it develops rapidly, is almost diagnostic of a heart-lesion -often of the onset of pericarditis.

Repeated bouts of sweating after the joints have settled down in the absence of heavy salicylate-dosage is very suggestive of heart-involvement.

Rashes-urticarial, macular or petechial unless of salicylate origin point to persistence of the rheumatic state and should call renewed attention to the heart.

Nodules in tendons or fascial attachments are always accompanied by vegetations on the endocardium.

If throughout the course of the illness i.e. during the whole of the essential six weeks in bed there has been no sign of cardiac disease, the temperature has been normal for three weeks, there is complete absence of pain and sweating, the child is feeling well and eating and sleeping well it may be said with confidence that the illness is over, that the heart is healthy and that after a month's convalescence with graduated exercise the child can safely return to work and play.

It is otherwise, however, if investigation of the points enumerated has at any time disclosed a cardiac lesion. Then an even more difficult question will arise. Is this lesion still active? The heart may be affected in one or both of two ways: there may be muscular changes leading to dilatation or there may be endocarditis. Pericarditis as such is of relatively little importance compared with the myocarditis which invariably accompanies it and may be included for our present purpose in the myocarditis group.

A myocardial lesion uncomplicated by a coincident endocarditis takes the form of weakening and dilatation and tends in a child to spontaneous and complete recovery: it occurs in this form during the early febrile stage of the illness and disappears without any specific treatment soon after the febrile stage is over. It is suggested by the appearance of a soft systolic murmur over the lower præcordium 
and a relative increase in the pulse-rate. Its danger is a tendency to recurrence within three months under conditions of physical strain and although it need not spell longer confinement to bed when recovery is apparently complete it does call for at least three months' convalescence and a very gradual return to full activity.

Endocarditis presents further problems. Three points have to be borne in mind: it is always associated with some degree of myocarditis with its tendency to dilatation under stress; vegetations may increase in size or become secondarily infected leading to progressive endocarditis with toxæmia and risk of emboli; or they may heal leaving in their train scarred valves whose mechanical handicap will in time produce gross heart-changes and shorten life. Such scarring is slow in development e.g. it probably takes two years for a definite mitral stenosis to establish itself. We are powerless to prevent it and from the point of view of controlling the individual case we need only seek evidence as to the persistence or disappearance of the actual vegetations. Here we can only conjecture and are guided by our estimate of the condition of the myocardium. So long as active endocarditis is present the signs of myocardial recovery detailed below will not be found, and there will be an ever present risk of dilatation occurring with corresponding increase in the heart's embarrassment. In these circumstances complete rest in bed must be maintained till there has been time for compensatory hypertrophy to take place: this cannot be expected in less than three months.

The occurrence of slight occasional rises of temperature to $99^{\circ}$ or $99.6^{\circ}$ need not by itself be taken as evidence of continuing heart-mischief - such irregularity of the temperature line demands scrupulous and repeated scrutiny of the case as a whole but if at the end of three months the general condition of the child is good, the pulse is comparable with the temperature, vagal rhythm is definite, appetite and sleep are normal the myocardium may be regarded as restored and it is permissible to let the child get up gradually and to institute a system of progressive activity under close supervision.

Such clemency is often rewarded by a disappearance of the irregular pyrexia and may prevent many months of unnecessary and depressing invalidism.
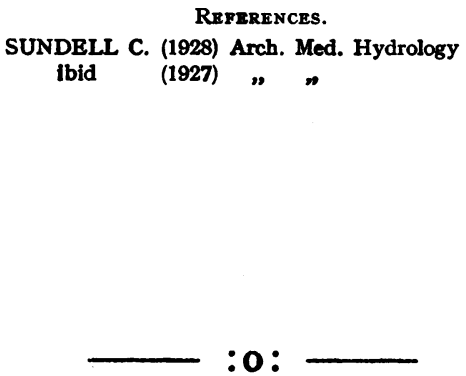